\title{
STUCK-AT-FAULT TESTABILITY OF SPP THREE-LEVEL LOGIC FORMS
}

\author{
Valentina Ciriani \\ Dip. di Tecnologie dell'Informazione \\ Universita' degli Studi di Milano, 26013 Crema, Italy \\ ciriani@dti.unimi.it
}

\author{
Anna Bernasconi \\ Department of Computer Science \\ University of Pisa, 56127 Pisa, Italy \\ annab@di.unipi.it
}

Rolf Drechsler

Institute of Computer Science

University of Bremen, 28359 Bremen, Germany

drechsler@informatik.uni-bremen.de

\begin{abstract}
Recently introduced, three-level logic Sum of Pseudoproducts (SPP) forms allow the representation of Boolean functions with much shorter expressions than standard two-level Sum of Products (SOP) forms, or other three-level logic forms. In this paper the testability of circuits derived from SPPs is analyzed. We study testability under the Stuck-At Fault Model (SAFM). For SPP networks several minimal forms can be considered. While full testability can be proved for some classes, others are shown to contain redundancies. Experimental results are given to demonstrate the efficiency of the approach.
\end{abstract}

Keywords: Reliability and Testing, Testability, Design for Testability, SPP Three-Level Network.

\section{Introduction}

An important aspect of logic synthesis is the problem of deriving highquality design from the initial specifications. A given Boolean function may be realized by a large variety of circuits, very different in terms of structure. In this framework the selection of a logic network, out of all possible known models (e.g., SOP [Coudert, 1994], ESOP [Koda and Sasao, 1995,

Please use the following format when citing this chapter:

Ciriani, Valentina, Bcrnasconi, Anna, Drechsler, Rolf, 2006, in IFIP International

Fcderation for Information Processing, Volume 200, VLSI-SOC: From Systems to

Chips, eds. Glesner, M., Reis, R., Indrusiak, L., Mooney, V., Eveking, H., (Boston:

Springer), pp. 299-313. 
Sasao, 1993, Sasao, 1996], EXSOP [Chattopadhyay et al., 1997, Debnath and Sasao, 1999, Dubrova et al., 1999], OR-AND-OR [Debnath and Vransic, 2003], SPP [Ciriani, 2003b, Luccio and Pagli, 1999], ESPP [Ishikawa et al,, 2002]), is critical and depends on multiple factors. Moreover it is very difficult to define a theoretical model that captures the problem in its generality. Thus the objective is to synthesize a circuit that optimizes a cost function involving different factors. In particular we are interested in several features like:

1 the size of the algebraic expression, in order to estimate the area occupied by the logic gates;

2 the number of levels in the network, in order to estimate the delay of the longest path through the gates;

3 the implementability of the network in the current technologies;

4 the existence of efficient minimization algorithms;

5 the testability properties of the network;

6 the power consuming of the network.

The standard synthesis is performed with Sum of Products (SOP) minimization procedures, leading to two-level circuits. More-than-two level minimization is much harder, but the size of the circuits can significantly decrease. In many cases three-level logic is a good trade-off among circuit speed, circuit size, and the time needed for the minimization procedure [Sasao, 1989]. Algorithms for exact minimization have worst case exponential complexity, hence the time to attain minimal forms may become huge for increasing size of the input.

In this paper we focus on a special three-level network called Sum of Pseudoproducts (SPP) and on the more general Sum of k-Pseudoproducts ( $k$-SPP). This choice is motivated by the fact that SPP networks often satisfy the above mentioned properties:

SPP expressions, introduced in [Luccio and Pagli, 1999], can be seen as a direct generalization of SOP expressions using EXOR gates. An SPP form consists of the OR of pseudoproducts, where a pseudoproduct is the AND of EXOR factors (i.e., EXOR of literals). In the recent paper [Ishikawa et al., 2002] a modified version of SPP networks, called ESPP and consisting of an EXOR of pseudoproducts, has been proposed. Among three-level networks, SPP forms are particularly compact [Ciriani, 2003a, Ciriani, 2003b]. However SPP forms have two major disadvantages: (i) they require large computational effort for the minimization; (ii) they have been originally defined for EXOR gates with unbounded fan-in, but in most technologies, EXOR gates with many inputs are slow, expensive and often not easily implementable [Weste and Eshraghian, 1993]. Therefore, in recent studies [Ciriani, 2003a, Ciriani, 2003b, Ciriani and Bernasconi, 2002], $k$-SPP forms with a fixed maximum number of literals $(k)$ in the EXOR factors have been introduced. 
Experimental results [Ciriani, 2003a, Ciriani, 2003b, Ciriani and Bernasconi, 2002] show that the size of the $k$-SPP minimal forms is not significantly larger than the one for unbounded fan-in, but the computational effort drastically decreases, especially when $k=2$. Thus, 2-SPP forms are reasonable upper bounds of the exact SPP forms, and are a good trade-off between the compactness of SPP forms and the efficiency of SOP minimization. Furthermore 2-SPP forms require a reduced number of different EXOR gates and are more practicable for the current technology. Moreover, preliminary results on multipliers indicate that SPP networks are also low power consuming [Ciriani et al., 2003].

Beside the synthesis aspect, testability is a major aspect of the design process. Up to $40 \%$ of the overall design costs are due to testing. For this, aspects of testability should be considered from the very beginning [Williams and Parker, 1982]. For several two-level forms detailed studies on testability have been performed. But, to the best of our knowledge, for three-level networks testability has not been considered so far.

In this paper the testability of 2-SPP and SPP forms is studied from a theoretical and practical point of view under the Stuck-At Fault Model (SAFM).

The classical stuck-at fault model (SAFM) is well-known and used throughout the industry [Breuer and Friedman, 1976]. In SAFM it is assumed that a defect causes a basic cell input or output to be fixed to either 0 or 1 . Thus, all failures with this effect will be detected by tests for stuck-at faults.

The investigations with respect to the SAFM are usually based on the single fault assumption, i.e., one assumes that there is at most one fault in the circuit. Under this model it is proved that general SPP networks, minimized with respect to the number of literals, are free of redundancies by construction. Whereas it can be shown by counter-examples that SPPs, minimized with respect to the number of products, are not fully testable. The same result holds for the specific class of 2-SPPs. Experimental results are given to demonstrate the efficiency of the approach.

The paper is structures as follows: In Section 2 notation and definitions are given. The stuck-at fault model is introduced and basics on SPP networks are reviewed. The testability results are presented in Section 3. In Section 4 details on the experimental setup and the practical results are given. Finally, the results are discussed in Section 5.

\section{Preliminaries}

\subsection{Stuck-at Fault Model (SAFM)}

Let $C$ be any combinational logic circuit over a fixed library. A fault in the SAFM [Breuer and Friedman, 1976] causes exactly one input or output pin of a node in $C$ to have a fixed constant value ( 0 or 1$)$ independently of the 
values applied to the primary inputs of the circuit. More precisely we have the following

Definition $1 \mathrm{~A}$ stuck-at fault with fault location $v$ is a tuple $(v[i], \epsilon)$ or $([i] v, \epsilon)$, where $v[i]([i] v)$ denotes the $i$-th input (output) pin of $v$, and $\epsilon \in\{0,1\}$ is the fixed constant value.

For brevity, in the following we simply speak of stuck-at- 0 or stuck-at-1 (s-a- 0 , $\mathrm{s}-\mathrm{a}-1)$ faults, if the context is clear.

DEFINITION 2 An input t to $C$ is a test for a fault $F$, iff the primary output values of $C$ on applying $t$ in the presence of $F$ are different from the output values of $C$ in the fault free case.

A fault is testable, iff there exists a test for this fault. The goal of any test pattern generation process is a complete test set for the circuit under test in the considered fault model, i.e. a test set that contains a test for each testable fault.

The construction of complete test sets requires the determination of the faults which are not testable ( $=$ redundant), even though it is easy to see that in general the detection of redundancies is coNP-complete. Redundancies have further unpleasant properties: they may invalidate tests for testable faults and often correspond to locations of the circuit where area is wasted [Breuer and Friedman, 1976]. For this, synthesis procedures which result in non-redundant circuits are desirable.

A node $v$ in $C$ is called fully testable, if there does not exist a redundant fault with fault location $v$. If all nodes in $C$ are fully testable, then $C$ is fully testable.

EXAMPLE 3 Consider the circuit in Figure 1. A s-a-0 fault at the output of the gate $\left(x_{1} \oplus x_{2}\right)$ can be tested by setting inputs $x_{3}$ and $x_{4}$ to 1 .

This is needed to ensure the propagation along the upper AND-gate. Since the EXOR of $x_{3}$ and $x_{4}$ then becomes 0 , the output of the lower AND-gate becomes also 0 , ensuring the propagation of the faulty value along the ORgate at the output. The test is independent of the value of input $x_{1}$.

\section{$2.2 \quad 2-S P P$ and SPP Networks}

In this section we recall some basic definitions from [Ciriani, 2003a, Ciriani, 2003b, Ciriani and Bernasconi, 2002].

In a Boolean space $\{0,1\}^{n}$ described by $n$ variables $x_{1}, x_{2}, \ldots, x_{n}$, a 2 EXOR factor is an EXOR with at most 2 variables, one of which possibly complemented (an EXOR with just one literal corresponds to the literal itself).

Given two Boolean variables $x_{1}, x_{2}$, all the possible 2-EXOR factors are essentially $x_{1}, \bar{x}_{1}, x_{2}, \bar{x}_{2},\left(x_{1} \oplus x_{2}\right)$ and $\left(x_{1} \oplus \bar{x}_{2}\right)$ (in fact, $\bar{x}_{1} \oplus x_{2}=x_{1} \oplus \bar{x}_{2}$, and $\left.\bar{x}_{1} \oplus \bar{x}_{2}=x_{1} \oplus x_{2}\right)$. 


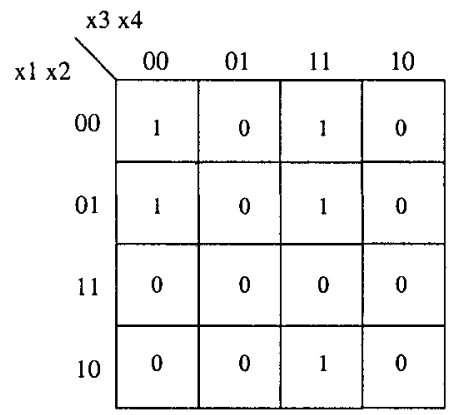

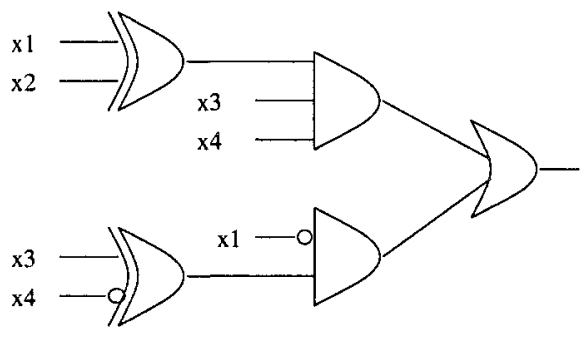

Figure 1. Karnaugh map of function $f$ with a 2-SPP cover $\left(x_{1} \oplus x_{2}\right) x_{3} x_{4}+\bar{x}_{1}\left(x_{3} \oplus \bar{x}_{4}\right)$, minimal with respect to the number of 2-pseudoproducts, and the corresponding 2-SPP circuit representation.

DEFINITION 4 A 2-pseudoproduct is a product of 2-EXOR factors, and a 2 SPP form is a sum of 2-pseudoproducts.

A 2-pseudoproduct $P$ of a Boolean function $f$ is prime iff no other 2pseudoproduct $P^{\prime}$ of $f$ exists such that $P \subseteq P^{\prime}$.

DEFINITION 5 A set of points whose characteristic function can be represented as a 2-pseudoproduct is a 2-pseudocube.

This is a generalization of the concept of cubes. A SOP form is a particular 2-SPP form where each EXOR factor contains only one literal.

In the space $\{0,1\}^{n}$ the number of different 2-EXOR factors with exactly 2 literals is $2 \cdot\left(\begin{array}{l}n \\ 2\end{array}\right)=n(n-1)$. Thus in the worst case, 2-SPP forms require a quadratic number of different 2-EXOR gates.

The 2-SPP synthesis problem can be stated as: given a set of points in the Boolean space $\{0,1\}^{n}$, find its minimal cover composed of 2-pseudocubes, where a minimal cover is represented by a sum of 2-pseudoproducts with a minimal number of literals or with a minimal number of 2-pseudoproducts.

EXAMPLE 6 For the function $f$ represented by the Karnaugh map in Figure 1, the following 2-SPP cover is a minimal expression with respect to 2pseudoproducts: $\left(x_{1} \oplus x_{2}\right) x_{3} x_{4}+\bar{x}_{1}\left(x_{3} \oplus \bar{x}_{4}\right)$.

The 2-SPP circuit representation is on the left side of the figure. On the other hand, a 2-SPP form minimal with respect to the number of literals is $\bar{x}_{2} x_{3} x_{4}+\bar{x}_{1}\left(x_{3} \oplus \bar{x}_{4}\right)$.

Finally, a minimal SOP form of such function is $\bar{x}_{2} x_{3} x_{4}+\bar{x}_{1} \bar{x}_{3} \bar{x}_{4}+\bar{x}_{1} x_{3} x_{4}$.

We can observe that a 2-pseudoproduct corresponds to a system of linear equations, and a 2-pseudocube corresponds to the set of solutions of such a system. 
EXAMPLE 7 The 2-pseudoproduct

$$
x_{2} \cdot\left(x_{1} \oplus x_{3}\right) \cdot\left(x_{3} \oplus \bar{x}_{5}\right) \cdot \bar{x}_{6} \cdot\left(x_{7} \oplus x_{8}\right)
$$

in $\{0,1\}^{9}$ corresponds to the system

$$
\left\{\begin{array}{r}
x_{2}=1 \\
x_{1} \oplus x_{3}=1 \\
x_{3} \oplus \bar{x}_{5}=1 \\
\bar{x}_{6}=1 \\
x_{7} \oplus x_{8}=1
\end{array}=\left\{\begin{array}{r}
x_{2}=1 \\
x_{1} \oplus x_{3}=1 \\
x_{3} \oplus x_{5}=0 \\
x_{6}=0 \\
x_{7} \oplus x_{8}=1
\end{array}\right.\right.
$$

When the 2-pseudocube is actually a cube, the system has only one variable in each equation.

A 2-pseudocube can be represented with different 2-pseudoproducts corresponding to different linear systems. For example, the three 2-pseudoproducts $x_{1} \cdot x_{1} \cdot\left(x_{2} \oplus x_{3}\right) \cdot\left(x_{2} \oplus x_{4}\right), x_{1} \cdot\left(x_{2} \oplus x_{3}\right) \cdot\left(x_{2} \oplus x_{4}\right) \cdot\left(x_{3} \oplus \bar{x}_{4}\right)$, and $x_{1} \cdot\left(x_{2} \oplus x_{3}\right) \cdot\left(x_{2} \oplus x_{4}\right)$ represent the same set of points (i.e., 2-pseudocube): $\{1011,1100\}$. Of course the most convenient representation is the third one.

The corresponding linear systems are:

$$
\left\{\begin{array}{r}
x_{1}=1 \\
x_{1}=1 \\
x_{2} \oplus x_{3}=1 \\
x_{2} \oplus x_{4}=1
\end{array}=\left\{\begin{array}{r}
x_{1}=1 \\
x_{2} \oplus x_{3}=1 \\
x_{2} \oplus x_{4}=1 \\
x_{3} \oplus x_{4}=0
\end{array}=\left\{\begin{array}{r}
x_{1}=1 \\
x_{2} \oplus x_{3}=1 \\
x_{2} \oplus x_{4}=1
\end{array}\right.\right.\right.
$$

Observe that only the third system has maximum rank, i.e. its equations are linearly independent, and indeed it corresponds to the smaller 2-pseudoproducts. Therefore minimal 2-SPP forms are sums of 2-pseudoproducts whose systems have maximum rank.

In [Ciriani and Bernasconi, 2002] a 2-SPP minimization algorithm is proposed. As in the Quine-McCluskey approach for the SOP forms, the generation of prime 2-pseudoproducts is performed in steps by successive unions of 2-pseudoproducts. A minimal 2-SPP form is generated by choosing a minimal subset of prime 2-pseudoproducts that covers the original function (this is the classical set covering step of Quine-McCluskey optimization).

The SPP forms, proposed and studied in [Ciriani, 2003a, Ciriani, 2003b, Luccio and Pagli, 1999], are a direct generalization of 2-SPP expressions, where the EXOR factors can have an unbounded number of literals.

\section{Testability in the SAFM}

In this section we study the testability of 2-SPP and SPP networks under the SAFM. As observed in Section 2.2 there exist two different notions of cost function for the minimization of 2-SPP (SPP) forms: 
1 The cost function is the total number of 2-pseudoproducts (pseudoproducts) in the form.

2 The cost function is the total number of literals in the form.

In both cases, the minimal forms are prime and irredundant. The full testability of 2-SPP and SPP forms is guaranteed only in the second case, as proved below, while forms minimized with respect to the number of pseudoproducts may contain redundancies.

\section{$3.1 \quad 2-S P P$ Networks}

We first consider 2-SPP forms minimal w.r.t. the number of 2-pseudoproducts.

THEOREM 82 2-SPP expressions minimal with respect to the number of 2pseudoproducts are not fully testable.

\section{Proof.}

We provide a counter-example. Consider the function $f=\{0101,0111$, $1001,1010,1101,1110\}$. There are three prime 2-pseudoproducts for $f:\left(x_{1} \oplus\right.$ $\left.x_{2}\right)\left(x_{3} \oplus x_{4}\right), x_{2}\left(x_{3} \oplus x_{4}\right)$, and $x_{1}\left(x_{3} \oplus x_{4}\right)$. The sum of any couple of them provides a 2-SPP form, prime and irredundant, and minimal w.r.t. the number of 2-pseudoproducts. In fact we have:

$$
\begin{aligned}
f & =\left(x_{1} \oplus x_{2}\right)\left(x_{3} \oplus x_{4}\right)+x_{2}\left(x_{3} \oplus x_{4}\right) \\
& =\left(x_{1} \oplus x_{2}\right)\left(x_{3} \oplus x_{4}\right)+x_{1}\left(x_{3} \oplus x_{4}\right) \\
& =x_{1}\left(x_{3} \oplus x_{4}\right)+x_{2}\left(x_{3} \oplus x_{4}\right) .
\end{aligned}
$$

Let us choose the form

$$
f=\left(x_{1} \oplus x_{2}\right)\left(x_{3} \oplus x_{4}\right)+x_{2}\left(x_{3} \oplus x_{4}\right) .
$$

Suppose that there is a s-a- 0 at the input $x_{2}$ of the gate $\left(x_{1} \oplus x_{2}\right)$. In this case the output of the 2-pseudoproduct $\left(x_{1} \oplus x_{2}\right)\left(x_{3} \oplus x_{4}\right)$ is identical to the output of $x_{1}\left(x_{3} \oplus x_{4}\right)$. Therefore the faulty network is equivalent to

$$
x_{1}\left(x_{3} \oplus x_{4}\right)+x_{2}\left(x_{3} \oplus x_{4}\right),
$$

that is exactly the original function $f$.

We now consider 2-SPP forms minimal w.r.t. the number of literals. We first need a preliminary result. Recall that 2-SPP networks are composed of three levels of logic: a level of 2-EXORs whose inputs are the variables; a level of ANDs whose inputs are the outputs of the EXOR layer; and an OR among the outputs of the AND layer. 
LEMMA 9 All possible values can be applied to the inputs of the AND layer of a minimal 2-SPP network.

Proof. Recall that a 2-pseudoproduct can be seen as a linear system. In a minimal 2-SPP form each 2-pseudoproduct contains a number of 2-EXOR factors equal to the rank of its system. In other words the equations in the corresponding system are linearly independent. This means that the outputs of the EXOR gates are independent, i.e., the inputs to the AND layer have all the possible values.

We can now prove the full testability of minimal 2-SPP networks.

THEOREM 10 2-SPP forms minimal with respect to the number of literals are fully testable.

Proof. Since 2-SPP forms are prime and irredundant, the proof of the full testability for AND and OR gates is the same as for SOP forms. As proved in Lemma 9, the inputs to the AND gates are directly controllable, i.e., all possible values can be applied. We are then left only with the case of s-afault at inputs of EXOR gates. We prove by contradiction that any fault can be tested.

Let $\left(x_{i} \oplus x_{j}\right) \cdot p+s$ be a representation of $f$ in 2-SPP form minimal w.r.t the number of literals, where $p$ is a 2-pseudoproduct and $s$ is the rest of the minimal 2-SPP form.

Let us consider the case $x_{i} \equiv 0$, i.e., s-a-0 in $x_{i}$. Then the network computes the faulty function $f_{F}=x_{j} \cdot p+s$. By contradiction suppose that $f_{F} \equiv f$, then

$$
\begin{aligned}
x_{j} \cdot p+s & \equiv\left(x_{i} \oplus x_{j}\right) \cdot p+s \\
x_{j} x_{i} \cdot p+x_{j} \bar{x}_{i} \cdot p+s & \equiv \bar{x}_{j} x_{i} \cdot p+x_{j} \bar{x}_{i} \cdot p+s \\
x_{j} x_{i} \cdot p+s & \equiv \bar{x}_{j} x_{i} \cdot p+s .
\end{aligned}
$$

Since $x_{j} x_{i} \cdot p \cap \bar{x}_{j} x_{i} \cdot p=\emptyset$, we have that

$$
x_{j} x_{i} \cdot p \subseteq s \text { and } \bar{x}_{j} x_{i} \cdot p \subseteq s,
$$

which implies that $x_{i} \cdot p \subseteq s$. Therefore $f$ contains $\left(x_{i} \oplus x_{j}\right) \cdot p$ and $x_{i} \cdot p$.

We now observe that

$$
x_{i} \cdot p+\left(x_{i} \oplus x_{j}\right) \cdot p=x_{i} \cdot p+x_{j} \cdot p .
$$

We have

$$
\begin{aligned}
x_{i} \cdot p+\left(x_{i} \oplus x_{j}\right) \cdot p & =x_{j} x_{i} \cdot p+\bar{x}_{j} x_{i} \cdot p+x_{j} \bar{x}_{i} \cdot p \\
& =x_{j} x_{i} \cdot p+\bar{x}_{j} x_{i} \cdot p+x_{j} x_{i} \cdot p+x_{j} \bar{x}_{i} \cdot p \\
& =x_{i} \cdot p+x_{j} \cdot p .
\end{aligned}
$$


Therefore we reach a contradiction to the minimality w.r.t. the number of literals of the 2-SPP form for $f$. The minimal 2-SPP form for $f$ would be $x_{j} \cdot p+s$ instead of $\left(x_{i} \oplus x_{j}\right) \cdot p+s$.

The case of negated variables is identical. An analogous proof holds for a s-a-1 fault.

\subsection{SPP Networks}

SPP networks have an unbounded number of literals in the EXOR gates. If we consider forms minimal w.r.t. the number of products, then we have the same result as for 2 -SPP networks, since the counter-example given in the proof of Theorem 8 still holds.

Consider now SPP forms minimal w.r.t. the number of literals. The result is analogous to the one for 2-SPP forms:

THEOREM 11 SPP forms minimal with respect to the number of variables are fully testable.

\section{Proof.}

Following the proof for 2-SPP forms we now have to prove the testability of general EXOR gates. Let $\left(x_{i} \oplus h\right) \cdot p+s$ be a representation of $f$ in SPP form minimal w.r.t the number of literals, where $h$ is an EXOR factor, not including $x_{i}, p$ is a pseudoproduct and $s$ is the rest of the minimal SPP form.

Let us consider the case $x_{i} \equiv 0$, i.e. s-a-0 in $x_{i}$. Then the network computes the faulty function $f_{F}=h \cdot p+s$. By contradiction suppose that $f_{F} \equiv f$, then

$$
\begin{aligned}
h \cdot p+s & \equiv\left(x_{i} \oplus h\right) \cdot p+s \\
h x_{i} \cdot p+h \bar{x}_{i} \cdot p+s & \equiv \bar{h} x_{i} \cdot p+h \bar{x}_{i} \cdot p+s \\
h x_{i} \cdot p+s & \equiv \bar{h} x_{i} \cdot p+s .
\end{aligned}
$$

Since $h x_{i} \cdot p \cap \bar{h} x_{i} \cdot p=\emptyset$, we have that

$$
h x_{i} \cdot p \subseteq s \text { and } \bar{h} x_{i} \cdot p \subseteq s
$$

which implies that $x_{i} \cdot p \subseteq s$. Therefore $f$ contains $\left(x_{i} \oplus h\right) \cdot p$ and $x_{i} \cdot p$. Observe that

$$
x_{i} \cdot p+\left(x_{i} \oplus h\right) \cdot p=x_{i} \cdot p+h \cdot p
$$

We have

$$
\begin{aligned}
x_{i} \cdot p+\left(x_{i} \oplus h\right) \cdot p & =h x_{i} \cdot p+\bar{h} x_{i} \cdot p+h \bar{x}_{i} \cdot p \\
& =h x_{i} \cdot p+\bar{h} x_{i} \cdot p+h x_{i} \cdot p+h \bar{x}_{i} \cdot p \\
& =x_{i} \cdot p+h \cdot p .
\end{aligned}
$$


Therefore we reach a contradiction to the minimality w.r.t. the number of literals of the SPP form for $f$.

Indeed a minimal form for $f$ would be $h \cdot p+s$ instead of $\left(x_{i} \oplus h\right) \cdot p+s$. An analogous proof holds for the s-a- 1 fault. 76

However, in practice SPP networks are defined once a variable ordering is fixed. In this case the above theorem, which refers to SPP forms minimal with respect to any possible variable ordering, does not hold any more. Moreover, as shown below, the SPP forms minimal w.r.t. a fixed variable ordering are no longer fully testable.

Let us consider minimal SPP forms depending on a variable ordering (for more details on SPP networks, see [Ciriani, 2003a, Ciriani, 2003b, Luccio and Pagli, 1999]). For example, consider the Boolean function $f=\{0011,0100$, $1000,1111\}$, and the variable ordering $o=x_{1}<x_{2}<x_{3}<x_{4}$.

The function $f$ is indeed a pseudocube, and its minimal SPP network, w.r.t. the variable ordering $o$, is $\left(x_{1} \oplus x_{2} \oplus x_{3}\right)\left(x_{1} \oplus x_{2} \oplus x_{4}\right)$. Meanwhile if we choose the variable ordering $x_{3}<x_{1}<x_{2}<x_{4}$, then a minimal SPP form is $\left(x_{3} \oplus x_{1} \oplus x_{2}\right)\left(x_{3} \oplus \bar{x}_{4}\right)$, which contains less literals than the former form.

In the case of 2-SPP networks, the number of literals in the minimal forms is instead independent of the variable ordering (see [Ciriani and Bernasconi, 2002] for more details); for this reason the testability theorem holds in any case.

If we fix an ordering, then the proof of testability given above cannot be applied anymore, as the following counter-example shows. Consider the function $f=\{00011,00100,00110,01001,01011,01110,10001,10011,10110$, $11011,11100,11110\}$. Once the variable ordering $o=x_{1}<x_{2}<x_{3}<x_{4}$ is fixed, there are eleven prime pseudoproducts for $f$.

A minimal form for $f$ in the variable ordering $o$ is:

$$
f=\left(x_{1} \oplus x_{2} \oplus x_{3} \oplus x_{4}\right)\left(x_{3} \oplus x_{5}\right)+x_{4}\left(x_{3} \oplus x_{5}\right) .
$$

Suppose that there is a s-a- 0 at the input $x_{4}$ of the gate $\left(x_{1} \oplus x_{2} \oplus x_{3} \oplus x_{4}\right)$. In this case the faulty function is:

$$
f_{F}=\left(x_{1} \oplus x_{2} \oplus x_{3}\right)\left(x_{3} \oplus x_{5}\right)+x_{4}\left(x_{3} \oplus x_{5}\right) .
$$

It is easy to verify that $f \equiv f_{F}$ but the pseudoproduct $p_{F}=\left(x_{1} \oplus x_{2} \oplus\right.$ $\left.x_{3}\right)\left(x_{3} \oplus x_{5}\right)$ is not represented in the order $o$. Therefore it is not in the set of eleven prime pseudoproducts used to form the minimal expression. In this case the fault cannot be detected because $f$ is indeed in minimal form w.r.t. the variable ordering $o$ and $f \equiv f_{F}$. Of course, if we do not fix a variable ordering then

$$
\left(x_{1} \oplus x_{2} \oplus x_{3} \oplus x_{4}\right)\left(x_{3} \oplus x_{5}\right)+x_{4}\left(x_{3} \oplus x_{5}\right)
$$

is not a minimal form for $f$. 
In summary, we get:

THEOREM 12 SPP forms minimal with respect to the number of literals in a fixed variable ordering are not fully testable.

\section{Experimental Results}

In this section experimental results for the SAFM are reported. The methods described above have been implemented in $\mathrm{C}$. The experiments have been run on a Pentium III $450 \mathrm{MHz}$ CPU with $128 \mathrm{MByte}$ of main memory.

The three-level forms have been optimized using the tools described in [Ciriani and Bernasconi, 2002] and the generated networks have been written as BLIF files. The verification of the correctness of the synthesis process and the testability analysis have been carried out in SIS [Sentovich et al., 1992]. The benchmarks are taken from LGSynth93 [Yang, 1991].

In a first series of experiments the quality of SPP forms (optimized by different criteria) are compared to two-level approaches. By this, an impression on the quality of the approaches is provided for a set of benchmarks. To this end we count the number of literals and gates (AND and EXOR) of an expression.

In the multi-level context the cost function is the total number of literals in all gates (see [Eggerstedt et al., 1993, Hachtel and Somenzi, 1996]). The problem is that in many technologies EXOR and OR (or AND) gates have different costs.

In [Hachtel and Somenzi, 1996] the authors consider a 2-input EXOR gate as $x \oplus y=x \cdot y+\bar{x} \cdot \bar{y}$. Thus the cost in literals of a 2-input EXOR gate is 4 , while the cost of the 2-input OR and AND gates is 2 . This is also proportional to the number of transistors used for the CMOS technology mapping (i.e., 4 transistors for AND/OR gates and 8 transistors for the EXOR gate).

More in general, by the associative property of the EXOR operator, we can always see a $k$-input EXOR gate as the composition of $(k-1) 2$-input EXOR gates. Therefore, we can use a function $\mu$ where a $k$-input EXOR gate costs $4(k-1)$, and $k$-input OR/AND gates cost $k$. This cost function corresponds to the CMOS cost described in [Eggerstedt et al., 1993].

Table 1 compares the costs of minimal 2-SPP, SOP and SPP forms (2-SPP and SPP networks are minimized with respect to the number of literals in the expressions). In the first column the name of the benchmark is given. In the next column the costs are given for 2-SPP, SOP and SPP forms. Here, $\mu$ is the cost for the 2-SPP network, while $\mu^{\prime}$ is the cost for the SOP network. The cost for the SPP network is $\mu^{\prime \prime}$. \#E is the number of different EXOR gates in 2-SPP and SPP forms. The star * indicates that the SPP algorithm did not terminate after 172800 seconds (corresponding to 2 CPU days).

The minimization algorithms are designed for exact synthesis of 2-SPP and SPP forms. Indeed the set of prime 2-pseudoproducts (pseudoproducts) is ex- 
Table 1. Costs for benchmark functions in 2-SPP, SOP and SPP forms

\begin{tabular}{l||r|r||r|r||r|r|r}
\hline \multicolumn{1}{l||}{} & \multicolumn{2}{c||}{ 2-SPP } & \multicolumn{2}{c||}{ SOP } & \multicolumn{3}{|c}{ SPP } \\
\hline name & \multicolumn{1}{l||}{$\mu$} & \#E & $\mu^{\prime}$ & $\mu / \mu^{\prime}$ & $\mu^{\prime \prime}$ & \multicolumn{1}{l|}{$\# \mathrm{E}$} & $\mu / \mu^{\prime \prime}$ \\
\hline$\overline{9 \text { sym }}$ & 168 & 18 & 588 & 0.29 & 188 & 30 & 0.89 \\
\hline addm4 & 694 & 34 & 1407 & 0.49 & $*$ & $*$ & $*$ \\
\hline adr4 & 105 & 5 & 415 & 0.25 & 118 & 10 & 0.89 \\
\hline clip & 402 & 26 & 769 & 0.52 & $*$ & $*$ & $*$ \\
\hline dist & 471 & 26 & 879 & 0.54 & 636 & 50 & 0.74 \\
\hline f51m & 232 & 19 & 402 & 0.58 & 243 & 23 & 0.95 \\
\hline life & 180 & 16 & 756 & 0.24 & 180 & 16 & 1.00 \\
\hline m4 & 735 & 28 & 1214 & 0.61 & 835 & 48 & 0.88 \\
\hline max512 & 620 & 35 & 1032 & 0.60 & $*$ & $*$ & $*$ \\
\hline mlp4 & 500 & 25 & 869 & 0.58 & 524 & 32 & 0.95 \\
\hline newcond & 161 & 11 & 239 & 0.67 & $*$ & $*$ & $*$ \\
\hline radd & 105 & 5 & 415 & 0.25 & 118 & 10 & 0.89 \\
\hline rd53 & 64 & 6 & 175 & 0.37 & 66 & 7 & 0.97 \\
\hline rd73 & 212 & 11 & 903 & 0.23 & 187 & 15 & 1.13 \\
\hline root & 281 & 21 & 376 & 0.75 & 366 & 31 & 0.77 \\
\hline squar5 & 101 & 6 & 120 & 0.84 & 112 & 8 & 0.90 \\
\hline xor5 & 24 & 2 & 96 & 0.25 & 18 & 1 & 1.33 \\
\hline z4 & 91 & 6 & 311 & 0.29 & 100 & 10 & 0.91 \\
\hline
\end{tabular}

actly computed. Since we used some heuristics [Fiorenzo-Catalano and Malucelli, 2001, Tebboth and Daniel, 2001] in solving the set covering problem, the number of literals in the expressions in Table 1 are upper bounds for the minimal solutions.

The corresponding minimization times are given in Table 2 . We note that 2-SPP and SPP forms are much more compact than the corresponding SOP expressions, 2-SPP minimization is also faster than SPP minimization with the exceptions of $9 s y m$ and $x o r 5$. This is due to the fact that the SPP minimization algorithm takes advantage of some regularities of functions (see [Bernasconi et al., 2003]), which cannot be exploited by the 2-SPP synthesis.

For all forms, the number of redundancies under the SAFM are given in Table 3. If SOPs are minimized, i.e. they are prime and irredundant, the corresponding networks are also fully testable. But compared to 2-SPP forms they are significantly larger in size (see above). Corresponding to the theoretical results in Section 3, it can be observed that 2-SPPs are fully testable in the SAFM (see Theorem 10), while SPPs may contain redundancies. Indeed the redundancies in SPP networks are due to the heuristic used for their synthesis, and to the fact that the variable ordering in the minimization algorithms is fixed (see Theorem 12). 
Table 2. Minimization times (in seconds)

\begin{tabular}{l||r||r||r}
\hline name & 2-SPP & SOP & SPP \\
\hline \hline 9sym & 242.67 & 5.32 & 147.58 \\
\hline addm4 & 50.96 & 0.87 & $*$ \\
\hline adr4 & 6.69 & 0.10 & 88.22 \\
\hline clip & 1662.27 & 0.38 & $*$ \\
\hline dist & 924.10 & 0.14 & 8196.00 \\
\hline f51m & 64.00 & 0.23 & 443.00 \\
\hline life & 120.40 & 0.03 & 262.00 \\
\hline m4 & 890.94 & 0.67 & 9929.40 \\
\hline max512 & 341.24 & 0.53 & $*$ \\
\hline mlp4 & 339.51 & 1.62 & 1423.74 \\
\hline newcond & 1485.01 & 0.01 & $*$ \\
\hline radd & 15.20 & 0.08 & 144.00 \\
\hline rd53 & 0.10 & 0.01 & 0.20 \\
\hline rd73 & 24.10 & 0.03 & 114.00 \\
\hline root & 272.32 & 0.08 & 1597.70 \\
\hline squar5 & 0.42 & 0.01 & 0.64 \\
\hline xor5 & 0.05 & 0.01 & 0.02 \\
\hline z4 & 5.30 & 0.04 & 6.75 \\
\hline
\end{tabular}

In summary, the experiments have shown that 2-SPP forms provide a very good compromise between compact representation, complexity of the minimization process and testability. Beside being more efficient than SOP regarding number of literals, they are so far the only three-level form that ensures full testability of the resulting circuit by construction.

\section{Conclusion}

Several approaches for three-level synthesis have recently been proposed. The resulting circuits have small delay but are more compact than two-level forms. The algorithmic complexity of the minimization algorithms are moderate. This makes them a promising candidate for synthesis.

In this paper we studied for the first time the testability of the resulting networks. For specific classes, i.e. 2-SPPs and SPPs minimal w.r.t. the number of literals in any variable ordering, full testability has been proved for the SAFM, while for other classes counter-examples were provided. Experimental results demonstrated the efficiency of the approach.

It is focus of current work to study more complex fault models, that allow to model dynamic behavior, like e.g. path-delay faults. 
Table 3. Number of redundancies

\begin{tabular}{l||r||r||r||r}
\hline name & original & 2-SPP & SOP & SPP \\
\hline \hline 9sym & 0 & 0 & 0 & 0 \\
\hline addm4 & 24 & 0 & 0 & $*$ \\
\hline adr4 & 24 & 0 & 0 & 0 \\
\hline clip & 0 & 0 & 0 & $*$ \\
\hline dist & 0 & 0 & 0 & 0 \\
\hline f51m & 56 & 0 & 0 & 0 \\
\hline life & 0 & 0 & 0 & 0 \\
\hline m4 & 22 & 0 & 0 & 3 \\
\hline max512 & 4 & 0 & 0 & $*$ \\
\hline mlp4 & 24 & 0 & 0 & 2 \\
\hline newcond & 0 & 0 & 0 & $*$ \\
\hline radd & 0 & 0 & 0 & 0 \\
\hline rd53 & 0 & 0 & 0 & 0 \\
\hline rd73 & 0 & 0 & 0 & 0 \\
\hline root & 0 & 0 & 0 & 1 \\
\hline squar5 & 12 & 0 & 0 & 1 \\
\hline xor5 & 0 & 0 & 0 & 0 \\
\hline z4 & 12 & 0 & 0 & 0 \\
\hline
\end{tabular}

\section{References}

[Bernasconi et al., 2003] Bernasconi, A., Ciriani, V., Luccio, F., and Pagli, L. (2003). ThreeLevel Logic Minimization Based on Function Regularities. IEEE Transactions on TCAD, 22(8):1005-1016.

[Breuer and Friedman, 1976] Breuer, M.A. and Friedman, A.D. (1976). Diagnosis \& reliable design of digital systems. Computer Science Press.

[Chattopadhyay et al., 1997] Chattopadhyay, S., Roy, S., and Chaudhuri, P. (1997). KGPMIN: An Efficient Multilevel Multioutput AND-OR-XOR Minimizer. IEEE Transaction on CAD, $16(3): 257-265$.

[Ciriani, 2003a] Ciriani, V. (2003a). Synthesis of SPP Three-Level Logic Networks using Affine Spaces. IEEE Transactions on TCAD, 22(10):1310-1323.

[Ciriani, 2003b] Ciriani, V. (2003b). Three-Level Logic Synthesis: Algebraic Approach and Minimization Algorithms. $\mathrm{PhD}$ thesis, Dipartimento di Informatica, University of Pisa.

[Ciriani and Bernasconi, 2002] Ciriani, V. and Bernasconi, A. (2002). 2-SPP: a Practical Trade-Off between SP and SPP Synthesis. In 5th International Workshop on Boolean Problems (IWSBP2002), pages 133-140.

[Ciriani et al., 2003] Ciriani, V., Luccio, F., and Pagli, L. (2003). Synthesis of Integer Multipliers in Sum of Pseudoproducts Form. Integration - the VLSI journal, 36(3):103-118.

[Coudert, 1994] Coudert, O. (1994). Two-Level Logic Minimization: an overview. INTEGRATION, 17:97-140. 
[Debnath and Sasao, 1999] Debnath, D. and Sasao, T. (1999). Multiple-Valued Minimization to Optimize PLAs with Output EXOR Gates. In IEEE International Symposium on MultipleValued Logic, pages 99-104.

[Debnath and Vransic, 2003] Debnath, D. and Vransic, Z.G. (2003). A Fast Algorithm for OR-AND-OR Synthesis. IEEE Transactions on Computer Aided Design, 22(9):1166-1176.

[Dubrova et al., 1999] Dubrova, E.V., Miller, D.M, and Muzio, J.C. (1999). AOXMIN-MV: A Heuristic Algorithm for AND-OR-XOR Minimization. In 4th Int. Workshop on the Applications of the Reed Muller Expansion in circuit Design, pages 37-54.

[Eggerstedt et al., 1993] Eggerstedt, M., Hendrich, N., and von der Heide, K. (1993). Minimization of Parity-Checked Fault-Secure AND/EXOR Networks. In IFIP WG 10.2 Workshop on Applications of the Reed-Muller Expansion in Circuit Design, pages 142-146.

[Fiorenzo-Catalano and Malucelli, 2001] Fiorenzo-Catalano, M. S. and Malucelli, F. (2001). Parallel Randomized Heuristics For The Set Covering Problem. International Journal of Computer Research, 10(4).

[Hachtel and Somenzi, 1996] Hachtel, G. and Somenzi, F. (1996). Logic Synthesis and Verification Algorithms. Kluwer Academy Publishers.

[Ishikawa et al., 2002] Ishikawa, R., Igarashi, T., Hirayama, T., and Shimizu, K. (2002). Pseudocube-based expressions to enhance testability. In IEEE Asia-Pacific Conference on Circuits and Systems, volume 2, pages 305-310.

[Koda and Sasao, 1995] Koda, N. and Sasao, T. (1995). An Upper Bound on the Number of Products in Minimum ESOPs. In IFIP WG 10.5 Workshop on Applications of the ReedMuller Expansions in Circuit Design.

[Luccio and Pagli, 1999] Luccio, F. and Pagli, L. (1999). On a New Boolean Function with Applications. IEEE Transactions on Computers, 48(3):296-310.

[Sasao, 1989] Sasao, T. (1989). On the Complexity of Three-Level Logic Circuits. In Int. Workshop on Logic Synthesis.

[Sasao, 1993] Sasao, T. (1993). AND-EXOR Expressions and their Optimization. In Sasao, T., editor, Logic Synthesis and Optimization. Kluwer Academic Publisher.

[Sasao, 1996] Sasao, T. (1996). Representation of Logic Functions Using EXOR Operators. In Sasao, T. and Fujita, M., editors, Representation of Discrete Functions. Kluwier Academic.

[Sentovich et al., 1992] Sentovich, E., Singh, K., Lavagno, L., Moon, Ch., Murgai, R., Saldanha, A., Savoj, H., Stephan, P., Brayton, R., and Sangiovanni-Vincentelli, A. (1992). SIS: A system for sequential circuit synthesis. Technical report, University of Berkeley.

[Tebboth and Daniel, 2001] Tebboth, J. and Daniel, R. (2001). A Tightly Integrated Modelling and Optimisation Library. Annals of Operations Research, 104:313-333.

[Weste and Eshraghian, 1993] Weste, N.H.E. and Eshraghian, K. (1993). Principles of CMOS VLSI Design. Addison-Wesley Publishing Company.

[Williams and Parker, 1982] Williams, T.W. and Parker, K.P. (1982). Design for Testability A Survey. IEEE Transactions on Computers, 31(1):2-15.

[Yang, 1991] Yang, S. (1991). Synthesis on Optimization Benchmarks. User guide, Microelectronic Center. Benchmarks available at $\mathrm{ftp}: / / \mathrm{ftp}$.sunsite.org.uk/computing/general/espresso.tar.Z. 